\title{
Randomised double blind trial of etoricoxib and indometacin in treatment of acute gouty arthritis
}

\author{
H Ralph Schumacher Jr, Judith A Boice, David I Daikh, Saurabh Mukhopadhyay, \\ Kerstin Malmstrom, Jennifer Ng, Guillermo A Tate, Javier Molina
}

Division of
Rheumatology,
University of
Pennsylvania
School of Medicine
and Department of
Veterans Affairs
Medical Center,
Philadelphia, PA
19104, USA
H Ralph
Schumacher Jr
professor of medicine
Department of
Clinical
Immunology and
Analgesia, Merck
Research
Laboratories,
Rahway, NJ 07065,
USA
Judith A Boice
senior medical
program coordinator
Kerstin Malmstrom
director
Department of
Clinical Biostatistics
and Research Data
Systems, Merck
Research
Laboratories
Saurabh
Mukhopadhyay
senior biometrician
Jennifer Ng
associate director
Division of
Rheumatology,
University of
California, San
Francisco and
Department of
Veterans Affairs
Medical Center, San
Francisco, CA
94121, USA
David I Daikh
assistant professor of
medicine
continued over

BMJ 2002;324:1488-92

\begin{abstract}
Objective To assess the safety and efficacy of etoricoxib, a selective cyclo-oxygenase-2 inhibitor, in comparison with indometacin in the treatment of acute gouty arthritis.
\end{abstract}

Design Randomised, double blind, active comparator controlled trial.

Setting 43 outpatient study centres in 11 countries. Participants 142 men and eight women (75 patients per treatment group) aged 18 years or over presenting with clinically diagnosed acute gout within 48 hours of onset.

Interventions Etoricoxib $120 \mathrm{mg}$ administered orally once daily versus indometacin $50 \mathrm{mg}$ administered orally three times daily, both for 8 days

Main outcome measures Patients' assessment of pain in the study joint over days 2 to 5 (primary end point); investigators' and patients' global assessments of response to treatment and tenderness of the study joint (key secondary end points).

Results Etoricoxib showed efficacy comparable to indometacin. Patients' assessment of pain in the study joint (0-4 point Likert scale, "no pain" to "extreme pain") over days 2 to 5 showed a least squares mean change from baseline of - 1.72 (95\% confidence interval -1.90 to -1.55$)$ for etoricoxib and -1.83 $(-2.01$ to -1.65$)$ for indometacin. The difference between treatment groups met prespecified comparability criteria. All other efficacy end points, including those reflecting reduction in inflammation and analgesia, provided corroborative evidence of comparable efficacy. Significant pain relief was evident at the first measurement, 4 hours after the first dose of treatment. Prespecified safety analyses revealed that drug related adverse experiences occurred significantly less frequently with etoricoxib $(22.7 \%)$ than with indometacin $(46.7 \%)(\mathrm{P}=0.003)$, although overall adverse experience rates were similar between the two treatment groups.

Conclusion Etoricoxib $120 \mathrm{mg}$ once daily provides rapid and effective treatment for acute gouty arthritis comparable to indometacin $50 \mathrm{mg}$ three times daily. Etoricoxib was generally safe and well tolerated in this study.

\section{Introduction}

Gout is the most common form of inflammatory joint disease in men over the age of $40 .{ }^{1}$ Acute gout is an intense, extremely painful, inflammatory arthritis with a rapidly escalating inflammatory response resulting from formation of monosodium urate crystals in the affected joint space. Although pain is the primary symptom of acute gout, an effective treatment must target both the pain and the underlying inflammation.
The drugs most often prescribed for acute gout are the non-steroidal anti-inflammatory drugs, of which indometacin is the standard treatment. ${ }^{23}$ Although indometacin is widely used, its efficacy is based on few studies, ${ }^{4-7}$ and it is associated with significant side effects in the gastrointestinal tract and central nervous system. ${ }^{89}$ Non-selective non-steroidal antiinflammatory drugs, including indometacin, inhibit two closely related enzymes-cyclo-oxygenase- 1 and cyclo-oxygenase-2. Cyclo-oxygenase-1 is broadly and constitutively expressed, whereas cyclo-oxygenase- 2 is an inducible enzyme involved in inflammatory processes. Cyclo-oxygenase-2 selective inhibitors, such as rofecoxib and celecoxib, have been shown to be as effective as non-steroidal anti-inflammatory drugs in the treatment of osteoarthritis, rheumatoid arthritis, and acute pain. ${ }^{10-14}$ However, the efficacy of selective cyclo-oxygenase-2 inhibitors in the treatment of acute inflammatory conditions such as acute gout has not been assessed.

Unlike other inflammatory conditions, such as those caused by tissue injury or aberrant immune responses, the inflammatory stimulus for acute gout is monosodium urate crystals. Although preclinical data have shown that cyclo-oxygenase-2 protein is induced in pro-inflammatory cells exposed to monosodium urate crystals, ${ }^{15}$ the rapid onset of a gout attack may indicate the involvement of the early response phase of inflammation, potentially involving cyclo-oxygenase- 1 . Analysis of synovial fluid from patients with acute gout supports the potential role of cyclo-oxygenase- $1 .{ }^{16}$ The efficacy of a cyclo-oxygenase-2 selective inhibitor in acute gout is therefore speculative in the absence of clinical data.

To determine whether selectively inhibiting cyclooxygenase- 2 in a rapid and sustained manner can effectively treat the pain and inflammation of acute gouty arthritis, we compared the efficacy of etoricoxib with that of indometacin.

\section{Methods}

\section{Study population}

Eligible patients were aged 18 years or over with acute gout (onset within 48 hours) associated with moderate, severe, or extreme pain and meeting the American Rheumatology Association diagnostic criteria for acute gout (see box on bmj.com). ${ }^{18}$ Additional criteria included baseline evaluations showing a sum score of $\geqslant 5$ for pain (0-4 point scale), tenderness (0-3 point scale), and swelling (0-3 point scale) and a complete blood count, blood chemistry, and urinalysis within one year before randomisation without clinically significant abnormalities or a normal complete blood count and serum creatinine obtained before dosing.

We excluded patients if they had acute polyarticular gout involving more than four joints; a concurrent 
medical or arthritic disease that could confound or interfere with the efficacy evaluation; or an unstable medical condition, including any history contraindicating the use of indometacin, a history of cancer during the previous five years, or a history of cerebrovascular events, myocardial infarction, or coronary bypass in the previous year. We also excluded patients taking corticosteroids within one month before randomisation or taking anticoagulants, ticlopidine, clopidogrel, or digoxin. We permitted patients to continue (without dose change) low dose aspirin $(\leqslant 325 \mathrm{mg}$ daily), allopurinol if taken for at least two weeks before randomisation, and colchicine ( $\leqslant 1.2 \mathrm{mg}$ daily) if taken at a stable dose for more than 30 days before randomisation. We did not allow non-steroidal antiinflammatory drugs within 48 hours before baseline assessments or analgesics, including aspirin, within six hours before baseline assessments and for the duration of the trial.

\section{Study design}

This was a randomised, double blind (the investigators and sponsor were blinded throughout the study), active comparator controlled study. The institutional review board or ethics review committee at each centre approved the protocol, and all patients gave written informed consent before participation. Patients were screened and, if eligible, randomised immediately (day 1), according to a computer generated allocation schedule provided by the sponsor, to receive oral administration of etoricoxib $120 \mathrm{mg}$ once daily or indometacin $50 \mathrm{mg}$ three times daily. Patients took one tablet of etoricoxib or placebo from bottle A once daily in the morning and one capsule of indometacin or placebo from bottle B three times daily (morning, afternoon, and evening). The treatment allocation was stratified for monoarticular or polyarticular acute gout. All patients completed pain assessments daily and returned to the clinic on days 2, 5, and 8 for assessment by the investigator; they also returned 14 days after the last dose for a post-study visit.

\section{Assessment of efficacy}

Efficacy end points included patients' assessment of pain in the study joint (primary end point) and investigators' assessments of tenderness, swelling, and erythema. We also evaluated patients' and investigators' global assessments of response to treatment.

The patients assessed pain in the study joint (0-4 point scale: "none," "mild," "moderate," "severe," "extreme") at baseline, four hours after the initial dose on day 1 , and approximately four hours after the first daily dose on days 2 to 8 . Investigators assessed tenderness of the study joint on the basis of palpation or passive motion (0-3 point scale: "no pain" to "patient states there is pain, winces, and withdraws"), swelling (0-3 point scale: "none" to "bulging beyond joint margins"), and erythema (present or absent) at baseline and at clinic visits. Patients' (0-4 point scale: "excellent" to "poor") and investigators' (0-4 point scale: "excellent" to "none") global assessments of response to treatment were collected at clinic visits. We also assessed the proportion of patients discontinuing treatment owing to lack of efficacy.
Assessment of safety and tolerability

Investigators performed physical examinations, assessed vital signs, and took samples for laboratory tests (complete blood count, blood chemistry, and urinalysis) at baseline and at the post-study visit. They also collected samples for laboratory tests on day 8 . They assessed vital signs and noted adverse experiences at all visits. Investigators evaluated all adverse experiences for intensity, seriousness, and relation to study drug while blinded to the treatment allocation.

\section{Statistical analysis}

We hypothesised that etoricoxib would show clinical efficacy comparable to indometacin as evaluated by patients' assessment of pain in the study joint (mean change from baseline over days 2 to 5 (primary end point) and over days 2 to 8 (secondary end point)). As prespecified in the data analysis plan, etoricoxib would be declared comparable to indometacin if the 95\% confidence interval for the between treatment difference fell within \pm 0.5 Likert units; these bounds were based on a consensus development (delphi) exercise in which \pm 0.5 was shown to be the minimum clinically important difference in osteoarthritis. ${ }^{19}$ The prespecified criteria for verifying the efficacy of indometacin required that the mean change from baseline in the indometacin group should be at least -1.46 Likert units. This criterion was based on a 95\% confidence interval for $60 \%$ of the predicted treatment effect $(-2.02$ to -1.46$)$, calculated using data from a study of indometacin versus ketoprofen ${ }^{4}$ and a non-treatment, observational study. ${ }^{20}$

On the basis of a standard deviation of 0.71 observed in a study of postorthopaedic surgical pain with rofecoxib, ${ }^{21} \mathrm{n}=62$ patients per group would have $95 \%$ power to show comparable efficacy if the true mean difference between etoricoxib and indometacin was zero. We analysed continuous efficacy variables by using a covariance model including factors for treatment, stratum, and baseline response. We obtained least squares means and associated 95\% confidence intervals to estimate and compare between treatment responses. We computed average responses from observed data and used the last value carried forward method for longitudinal graphs. We used Fisher's exact test and the Wilson's score method to compare variables analysed as proportions, including analysis of prespecified adverse experiences. ${ }^{22}$ We based analyses on an intention to treat approach (all patients with a baseline and at least one on-treatment measurement).

\section{Results}

\section{Characteristics of the patients}

Forty three study centres (hospital clinics, urgent care centres, and office practices) in 11 countries participated. A total of 31 centres enrolled 150 patients ( 75 in each group) between June and December 2000. Figure A on bmj.com shows a schematic representation of patient accounting throughout the study. Baseline characteristics (table A on bmj.com) and compliance rates were generally similar in both groups (around $91 \%)$.

\section{Efficacy}

Etoricoxib and indometacin showed comparable efficacy in the treatment of acute gouty arthritis as
Centro de

Osteopatias, Reumatologia e Investigaciones Clinicas, 1012 Buenos Aires, Argentina

Guillermo A Tate

director

Department of Internal Medicine, Antioquia

University, Calle 64 Carrera $51 \mathrm{D}$,

Medellin-Colombia 1226

Javier Molina honorary professor of medicine

Correspondence to: J A Boice judith_boice@ merck.com 


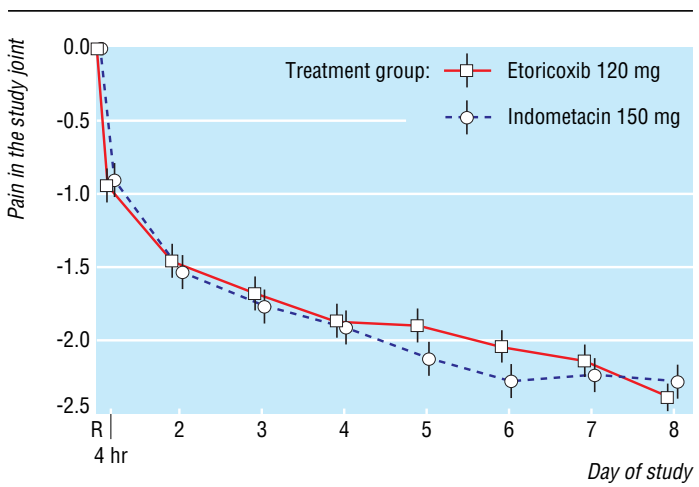

Fig 1 Least squares mean and standard error for the change from baseline in patients' assessment of pain in the study joint (primary end point) over days 2 to 8 based on a $0-4$ point Likert scale with $0=$ no pain, $1=$ mild pain, $2=$ moderate pain, $3=$ severe pain, and $4=$ extreme pain. The four hour time point indicates the assessment four hours after the initial dose of study drug on day 1 , the day of the randomisation $(\mathrm{R})$ visit (baseline)

assessed by all efficacy end points over the first four days (days 2 to 5 ) and over the entire treatment period (days 2 to 8) (fig 1). Furthermore, indometacin showed efficacy consistent with the predefined treatment effect $(-1.83 ; 95 \%$ confidence interval -2.01 to -1.65$)$.

\section{Primary end point}

The mean (SE) baseline values for patients' assessment of pain in the study joint (primary end point) were 2.88 (0.08) units for etoricoxib and $2.99(0.07)$ units for indometacin. Mean changes from baseline at each time point (fig 1) reveal that both treatment groups experienced comparable pain relief over the entire treatment period. The least squares mean difference between etoricoxib and indometacin for change in pain in the study joint from baseline was 0.11 (95\% confidence interval -0.14 to 0.35 ) over days 2 to 5 and 0.09 $(-0.14$ to 0.33$)$ over days 2 to 8 . Thus for both time periods the difference between treatments was within the prespecified boundaries for comparability. On day 1 , four hours after the initial dose, patients in both groups experienced significant pain relief (fig 1). The between group treatment effect was similar-least squares mean difference between the two treatment groups of -0.03 ( -0.27 to 0.21$)$. Improvements on days 2 and 3 were also robust and comparable between treatment groups-least squares mean change from baseline of -1.45 and -1.52 (day 2) and -1.66 and -1.76 (day 3) for etoricoxib and indometacin. The mean change from baseline criterion of -1.46 Likert units for indometacin, although not prespecified for these time periods, was also met.

Analysis of the primary end point included 72/75 patients in the etoricoxib group and $71 / 75$ patients in the indometacin group. Seven patients were not included in this analysis owing to lack of any pain assessments from days 2 to 5 .

Secondary end points

Etoricoxib showed efficacy similar to indometacin for all secondary efficacy end points (global assessments of response to treatment, joint tenderness and swelling (table 1), and discontinuation owing to lack of efficacy) and erythema. Five patients, three on etoricoxib and two on indometacin, discontinued owing to lack of efficacy $(\mathrm{P}>0.200)$. The proportion of patients with erythema in the study joint at baseline was high in both groups-89.3\% for etoricoxib and $93.3 \%$ for indometacin-and decreased similarly over the entire treatment period: to $12.2 \%$ for etoricoxib and $13.7 \%$ for indometacin on day 8 . The between treatment difference was similar at all measured time points (not shown)

\section{Onset of efficacy}

Approximately $70 \%$ of patients experienced severe or extreme pain at baseline (see table A on bmj.com). We performed a retrospective analysis of the proportion of patients in each treatment group who reported mild pain or no pain at four hours after initial dosing and on days 2,5 , and 8 to provide an additional clinically meaningful measure of pain relief. Although the results of this analysis were similar in the two treatment groups (fig 2), we noted that $32 \%$ of the etoricoxib patients and 23\% of the indometacin patients reported

Table 1 Summary of secondary end points for days 2 to 8

\begin{tabular}{|c|c|c|c|c|}
\hline & $\begin{array}{l}\text { Baseline } \\
\text { (mean) }\end{array}$ & $\begin{array}{l}\text { Treatment period } \\
\text { (mean) }\end{array}$ & $\begin{array}{c}\text { Least squares mean change from } \\
\text { baseline }(95 \% \mathrm{Cl})\end{array}$ & $\begin{array}{l}\text { Least squares mean difference } \\
\text { from indometacin }(95 \% \mathrm{Cl})^{\star *}\end{array}$ \\
\hline \multicolumn{5}{|c|}{ Tenderness of study joint (0-3 scale) $)^{\star} \dagger$} \\
\hline Etoricoxib $120 \mathrm{mg}(\mathrm{n} / \mathrm{N}=74 / 75)$ & 2.51 & 0.72 & $-1.76(-1.91$ to -1.62$)$ & $-0.01(-0.22$ to 0.20$)$ \\
\hline Indometacin $150 \mathrm{mg}(\mathrm{n} / \mathrm{N}=73 / 75)$ & 2.49 & 0.70 & $-1.75(-1.91$ to -1.60$)$ & NA \\
\hline \multicolumn{5}{|l|}{ Swelling of study joint (0-3 scale) ${ }^{\star} \ddagger$} \\
\hline Etoricoxib $120 \mathrm{mg}(\mathrm{n} / \mathrm{N}=74 / 75)$ & 2.28 & 0.87 & $-1.45(-1.61$ to -1.29$)$ & $0.00(-0.22$ to 0.23$)$ \\
\hline Indometacin $150 \mathrm{mg}(\mathrm{n} / \mathrm{N}=73 / 75)$ & 2.52 & 0.97 & $-1.45(-1.62$ to -1.28$)$ & NA \\
\hline \multicolumn{5}{|c|}{ Patients' global assessment of response to treatment (0-4 Likert scale)§ } \\
\hline Etoricoxib $120 \mathrm{mg}(\mathrm{n} / \mathrm{N}=74 / 75)$ & NA & 1.36 & $1.42(1.20$ to 1.65$)$ & $0.10(-0.21$ to 0.41$)$ \\
\hline Indometacin $150 \mathrm{mg}(\mathrm{n} / \mathrm{N}=72 / 75)$ & NA & 1.20 & $1.33(1.10$ to 1.56$)$ & NA \\
\hline \multicolumn{5}{|c|}{ Investigators' global assessment of response to treatment (0-4 Likert scale) ${ }^{\star} \pi$} \\
\hline Etoricoxib $120 \mathrm{mg}(\mathrm{n} / \mathrm{N}=74 / 75)$ & NA & 0.83 & 0.89 (0.70 to 1.08$)$ & $0.01(-0.25$ to 0.28$)$ \\
\hline Indometacin $150 \mathrm{mg}(\mathrm{n} / \mathrm{N}=73 / 75)$ & NA & 0.79 & 0.88 (0.69 to 1.08$)$ & NA \\
\hline
\end{tabular}

$\mathrm{NA}=$ not applicable; $\mathrm{n} / \mathrm{N}=$ total number of patients in analysis $(\mathrm{n})$ versus total number of patients randomised to treatment group (N). (Total number of patients included in analysis for each end point $(\mathrm{n})$ is based on number of patients with baseline score and at least one post-treatment score.) ${ }^{*}$ All investigator assessments throughout the study were carried out by the same physician for a given patient.

$+0=$ no pain; $1=$ patient states that there is pain; $2=$ patient states that there is pain and winces; $3=$ patient states that there is pain, winces, and withdraws. $\neq 0=$ none; $1=$ palpable; $2=$ =visible; $3=$ bulging beyond joint margins.

$\S 4=$ poor; $3=$ fair; $2=$ good; $1=$ very good; $0=$ excellent.

१4=none-no response, absence of drug effect; $3=$ poor—minimal response, unacceptable; $2=$ definite response, but could be better; $1=$ good—good response, but less than best possible anticipated result; $0=$ excellent-best possible anticipated response, considering severity of gout attack.

**Patient and investigator global assessments of response to treatment are least squares mean treatment values. 
no pain or mild pain within four hours of dosing. Of the patients who reported severe or extreme pain at baseline, $22 \%$ in the etoricoxib group and $19 \%$ in the indometacin group had mild or no pain within four hours of dosing.

\section{Consistency of efficacy across disease types}

The reduction of pain in the study joint was consistent between monoarticular and polyarticular gout $(\mathrm{P}>0.200)$ and across subgroups defined by concomitant use of allopurinol, colchicine, or both.

\section{Safety}

Four serious adverse experiences were reported, all in the indometacin group: vomiting and headache (determined by the investigator to be drug related), a drug overdose, and a laryngeal neoplasm (determined not to be drug related). For all four prespecified categories of adverse experiences, etoricoxib was associated with a lower incidence than indometacin (table 2). Drug related adverse experiences showed a significant difference $(\mathrm{P}=0.003)$; the most disparate were dizziness ( 4 patients in the etoricoxib group and 15 in the indometacin group), headache (1 and 5), somnolence (1 and 4$)$, and a variety of digestive adverse experiences (6 and 17). Five patients in the etoricoxib group and six in the indometacin group had one or more laboratory finding that was considered to be an adverse experience.

\section{Discussion}

Previous work has shown that cyclo-oxygenase-2 selective inhibitors provide efficacy similar to that of non-selective non-steroidal anti-inflammatory drugs in chronic inflammatory conditions and acute pain. However, the precise roles of cyclo-oxygenase- 1 and cyclooxygenase- 2 in acute inflammatory conditions such as acute gout have not been studied. In this study, we found that etoricoxib, which rapidly and selectively inhibits cyclo-oxygenase-2, had efficacy comparable to that of indometacin, the standard treatment for acute gout. We characterised the efficacy of etoricoxib on a wide variety of clinical manifestations of gout, including measurements that assessed both pain and

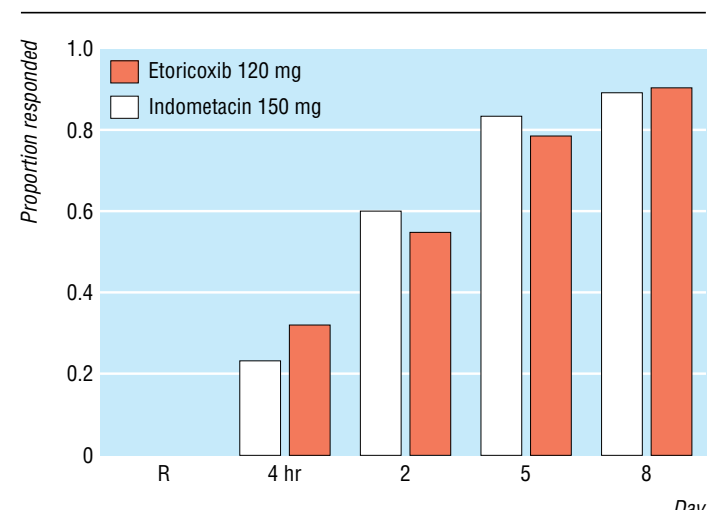

Fig 2 Proportion of clinically meaningful responses for patients' assessment of pain in the study joint for all patients in both treatment groups. Response=patients' assessment of pain is "mild" or "none;" R=randomisation visit; $4 \mathrm{hr}=$ four hours after the initial dose on day 1
Table 2 Analysis of prespecified adverse experiences. Values are numbers (percentages) of patients unless stated otherwise

\begin{tabular}{lccc} 
Type of adverse experience & $\begin{array}{c}\text { Etoricoxib } \\
(\mathbf{n}=\mathbf{7 5 )}\end{array}$ & $\begin{array}{c}\text { Indometacin } \\
(\mathbf{n}=\mathbf{7 5 )}\end{array}$ & P value $^{*}$ \\
\hline One or more clinical adverse experiences & $35(46.7)$ & $45(60.0)$ & 0.141 \\
\hline Drug related clinical adverse experiences† & $17(22.7)$ & $35(46.7)$ & 0.003 \\
\hline Serious clinical adverse experiences $\ddagger$ & 0 & $3(4.0)$ & 0.245 \\
\hline Discontinued owing to clinical adverse experiences & $2(2.7)$ & $8(10.7)$ & 0.098 \\
\hline
\end{tabular}

*Fisher's exact test.

†Determined by the investigator to be possibly, probably, or definitely drug related.

fDrug related vomiting and headache (1 patient), non-drug related laryngeal neoplasm (1), non-drug related drug overdose (1).

inflammation, and found the efficacy of etoricoxib to be comparable to that of indometacin. Etoricoxib provided pain relief comparable to indometacin as early as four hours after the initial dose, the first time point assessed. The proportion of patients reporting mild or no pain at the initial time point showed the powerful and rapid analgesic and anti-inflammatory effect of both drugs. Our results-in combination with the established pharmacology of etoricoxib ${ }^{23}$ - provide strong evidence in support of the hypothesis that selective inhibition of cyclo-oxygenase- 2 alone is sufficient to treat the pain and inflammation of acute gout.

Few clinical trials have been performed in acute gout, and these trials enrolled small numbers of patients, so there is only suggestive evidence of the effectiveness of various treatments. ${ }^{24}$ This study, representing the largest controlled trial in acute gout reported to date, had a rigorous design. Patients satisfied established diagnostic criteria to ensure that they were having an attack of gout and were enrolled within 48 hours of the onset of the attack. The primary efficacy assessment was over the first four days. Given the self limiting nature of acute gout, this design ensured that the emphasis was on the initial days of an attack, the period least likely to be influenced by spontaneous improvements that could skew the efficacy analysis. The end points chosen had been used in previous studies on acute gout but, owing to the paucity of previous studies on acute gout, are only validated in studies of osteoarthritis and analgesia.

In this study etoricoxib and indometacin were generally safe and well tolerated. Even this large study was still too small to allow for a rigorous safety assessment. The data on drug related clinical adverse experiences show that etoricoxib may be better tolerated than indometacin, but additional studies are needed to enable any definitive conclusions on safety to be drawn.

In conclusion, these results showed that etoricoxib had comparable efficacy to indometacin, proving that selective, potent, and rapid inhibition of cyclooxygenase-2 is sufficient to treat acute gouty arthritis effectively. The demographics of the patients enrolled in this study were typical of patients with acute gout, and this, together with the diversity of the study sites, means that these results are likely to be applicable to patients with acute gout in general. The results of this study indicate that etoricoxib represents an effective treatment alternative to indometacin.

We thank Elliot Ehrich for invaluable contributions to the design of the study, Peter Merkel for helpful suggestions regarding the study design, and Ken Truitt for guidance during the course of the study. We also thank Briggs Morrison, Ned Braunstein, and Barry Gertz for their helpful comments and suggestions. The Acute Gout Study Group consisted of the 


\section{What is already known on this topic}

Acute gouty arthritis is an extremely painful inflammatory arthritis resulting from deposition of monosodium urate in the affected joint space

The most common treatment is a non-selective non-steroidal anti-inflammatory drug, with indometacin being the standard treatment

Current treatment modalities are based on limited, small studies

\section{What this study adds}

Etoricoxib, a cyclo-oxygenase-2 selective inhibitor, has comparable efficacy to indometacin in the treatment of acute gouty arthritis

Etoricoxib provides rapid relief of pain and effectively treats the inflammation of acute gout; it is an effective treatment alternative to indometacin

following investigators: G Tate (Argentina); J Bentin (Belgium); R Fuller, D Souza (Brazil); J Basualdo (Chile); J Londono, J Molina (Colombia); V Thouas (Greece); J Orozco Alcala, J Vasquez-Mellado (Mexico); L McLean (New Zealand); J Antigua, S Navarra, E Osio-Salido (Philippines); A Lubbe, H Reuter (South Africa); W Blackburn, M Brown (AL, USA); D Arkfeld, D I Daikh, R Kaplan, A Kavanaugh, P Stein (CA, USA); G Gladstein (CT, USA); M Wooten (DE, USA); P Saxe, J Valeriano (FL, USA); C Agudelo, D Conn (GA, USA); T Schnitzer (IL, USA); D Neustadt (KY, USA); P Holt, P Merkel (MA, USA); P Bonafede, H W Emori (OR, USA); G Gordon, H R Schumacher Jr (PA, USA); J Aelion, K Lohr (TN, USA); C Jackson (UT, USA); R Bettis (WA, USE); G Kerr (Washington DC, USA); L Warrick (WI, USA).

Contributors: HRS contributed to the design and recruitment of the study. JAB contributed to the design and clinical aspects of the study and is the guarantor. DID contributed to the design and recruitment of the study. SM analysed the data. KM contributed to the clinical aspects of the study. JN participated in the design of the study. GAT and JM contributed to the recruitment of the study. All authors contributed to the writing of the paper.

Funding: Merck Research Laboratories, Rahway, NJ, USA, provided funding to all participating investigators to cover the costs of patient procedures and evaluations.

Competing interests: HRS is on the Merck arthritis advisory board and has received fees for speaking and organising educational programmes and consultancy fees from Merck Research Laboratories and Pfizer. DID has received fees for speaking and a consultancy fee from Merck Research Laboratories. JAB, SM, KM, and JN are employed by Merck and own shares of Merck common stock.

1 Roubenoff R. Gout and hyperuricemia. Rheum Dis Clin North Am 1990;16:539-50
2 Shrestha M, Morgan D, Moreden, J, Singh R, Nelson M, Hayes J Randomized double-blind comparison of the analgesic efficacy of intramuscular ketorolac and oral indomethacin in the treatment of acute gouty arthritis. Ann Emerg Med 1995;26:682-6.

3 Pittman JR, Bross MH. Diagnosis and management of gout. Am Fam Phy sician 1999;59:1799-806.

4 Altman RD, Honig S, Levin JM, Lightfoot RW. Ketoprofen versus indomethacin in patients with acute gouty arthritis: a multicenter, double blind comparative study. J Rheumatol 1988;15:1422-6.

5 Axelrod D, Preston S. Comparison of parenteral adrenocorticotropic hormone with oral indomethacin in the treatment of acute gout. Arthritis Rheum 1988;31:803-5.

6 Lomen PL, Turner LF, Lamborn KR, Winblad MA, Sack RL, Brinn EL. Flurbiprofen in the treatment of acute gout: a comparison with indomethacin. Am J Med 1986;80:134-9.

7 Ruotsi A, Vainio U. Treatment of acute gouty arthritis with proquazone and indomethacin. Scand J Rheumatol 1978;21:15-7.

8 Singh G, Rosen-Ramey D. NSAID induced gastrointestinal complications: the ARAMIS perspective-1997.J Rheumatol 1998;51:8-16.

9 Thompson M, Percy JS. Further experience with indomethacin in the treatment of rheumatic disorders. BMJ 1966; i:80-3.

10 Cannon GW, Caldwell JR, Holt P, McClean B, Seidenberg B, Bolognese J, et al for the Rofecoxib Phase III Protocol 035 Study Group. Rofecoxib, a specific inhibitor of cyclooxygenase 2 , with clinical efficacy comparable with that of diclofenac sodium: results of a one-year, randomized, clinical trial in patients with osteoarthritis of the knee and hip. Arthritis Rheum 2000;43:978-87.

11 McKenna F, Borenstein D, Wendt H, Wallenmark C, Lefkowitz JB, Geis GS. Celecoxib versus diclofenac in the management of osteoarthritis of the knee. Scand J Rheumatol 2001;30:11-8.

12 Emery P, Zeidler H, Kvien TK, Guslandi M, Naudin R, Stead H, et al. Celecoxib versus diclofenac in long-term management of rheumatoid arthritis: randomised double-blind comparison. Lancet 1999;354:2106-11.

13 Malmstrom K, Daniels B, Kotey P, Seidenberg BC, Dejardines PJ. Comparison of rofecoxib and celecoxib, two cyclooxygenase-2 inhibitors, in postoperative dental pain: a randomized, placebo- and activecomparator-controlled clinical trial. Clin Ther 1999:21:1653-63.

14 Gimbel JS, Brugger A, Zhao W, Verburg KM, Geis GS. Efficacy and tolerability of celecoxib versus hydrocodone/acetaminophen in the treatment of pain after ambulatory orthopedic surgery in adults. Clin Ther 2001;23:228-41

15 Pouliot M, James MJ, McColl SR, Naccache PH, Cleland LG. Monosodium urate microcrystals induce cyclooxygenase-2 in human monocytes. Blood 1998;91:1769-76.

16 Iniguez MA, Pablos JL, Carreira PE, Cabre F, Gomez-Reino JJ. Detection of COX-1 and COX-2 isoforms in synovial fluid cells from inflammatory joint diseases. Br J Rheumatol 1998;37:773-8.

17 Wallace SL, Robinson H, Masi AT, Decker JL, McCarty DJ, Yü T. Preliminary criteria for the classification of the acute arthritis of primary gout Arthritis Rheum 1977;20:895-900

$18 \mathrm{KlippelJH,} \mathrm{ed.} \mathrm{Primer} \mathrm{on} \mathrm{the} \mathrm{rheumatic} \mathrm{diseases:criteria} \mathrm{for} \mathrm{the} \mathrm{classification} \mathrm{of}$ acute gouty arthritis. Atlanta, USA: Arthritis Foundation, 1997:457.

19 Bellamy N, Carette S, Ford PM, Kean WF, le-Riche NG, Lussier A, et al. Osteoarthritis antirheumatic drug trials. III. Setting the delta for clinical trials-results of a consensus development (delphi) exercise. J Rheumatol 1992;19:451-7.

20 Bellamy N, Downie WW, Buchanan WW. Observations on spontaneous improvement in patients with podagra: implications for therapeutic trials of non-steroidal anti-inflammatory drugs. Br J Clin Pharmacol 1987; 24:33-6.

21 Reicin A, Brown J, Jove M, Andrade JR, Krupa D, Walters D, et al. Efficacy of single-dose and multidose rofecoxib in the treatment of postorthopedic surgery pain. Am J Orthop 2001,30:40-8.

22 Newcombe RG. Interval estimation for the difference between independent proportions: comparison of eleven methods. Stat Med 1998;17: $873-90$

23 Agrawal N, Porras AG, Matthews CZ, Woolf EJ, Miller JL, Mukhopadhyay $\mathrm{S}$, et al. Dose proportionality of oral etoricoxib, a highly selective cyclooxygenase-2 inhibitor, in healthy volunteers. I Clin Pharmacol 2001:41:1106-10.

24 Schlesinger N, Baker DG, Schumacher HR Jr. How well have diagnostic tests and therapies for gout been evaluated? Curr Opin Rheumatol 1999;11:441-5.

(Accepted 11 April 2002)

\section{A memorable patient}

\section{By any other name}

An elderly lady-"old" to me at the time--was pushed into my consulting room in a wheelchair by a caring relative, whom I thanked for her help. The patient had had diabetes for many years, an amputation of one leg, and a recent cardiac infarct. Unfortunately, she also had a severe visual handicap (no patient is ever "blind," at least in their hearing) because of advanced diabetic retinopathy. At the end of the consultation, I held the patient's hand and sympathetically explained that I was very sorry not to be able to offer any treatment for her reduced vision and that it was due to the general disease, diabetes, which she had. Whereupon she reared up as well as she could, agitated, and exclaimed, "I haven't got a disease, have I, doctor?"

Thereafter, I only ever spoke of a condition or similar word to a patient or relative. And, of course, no one should ever do double blind trials, only double masked ones.

Calbert I Phillips retired ophthalmologist, Edinburgh 